\title{
A Novel Pulse Compression Technique Using Inverse Filtering in Frequency Domain
}

\author{
G. S. Jeng, S. Huang, P. -C. Li and J. Tsao \\ Department of Electrical Engineering, National Taiwan University, Taipei, Taiwan, R.O.C.
}

Abstract - A pulse compression technique using inverse filtering in frequency domain to reduce range sidelobes is proposed. When frequency-dependent attenuation is taken into account, the achievable range sidelobes associated with conventional time-domain depth-independent pulse compression methods are usually unacceptable. In contrast, as long as the truncated echoes present at the beginning of received data can be removed, the range sidelobes for the proposed method can still be controlled to a lower level by increasing the data recording time. Both simulations and experiments are performed to demonstrate the efficacy of the method and suggest that chirp excitation fits it well.

\section{INTRODUCTION}

In order to improve signal-to-noise ratio (SNR) with limited acoustic peak pressure, coded excitations with pulse compression schemes have been widely studied in medical ultrasound. While SNR improvement can be achieved by emitting an elongated pulse, the performance of the code excitation system, especially contrast resolution, is mainly determined by range sidelobes resulting from the pulse compression. A variety of pulse compression methods have been proposed in [1] [4]. They are all realized in time domain and perform linear convolutions. Therefore, there still exists a limitation on achievable range sidelobe level due to the finite length of the compression filter. When frequency-dependent attenuation is taken into account, the compression result degrades gradually as the depth increases [3], [4]. Introducing a depth-dependent filter can solve this problem as long as attenuation coefficient is known in some way [3].

In this paper, an approach dismissing attenuation estimation is considered. The proposed pulse compression scheme employs the inverse filter and a proper shaping window, all implemented in frequency domain. The resultant range sidelobes can be extremely low if echoes from targets are not truncated. Due to limited recording time, however, incomplete echoes are present at the ends of the received data. As a result, compressing these incomplete echoes might cause high range sidelobes over the entire region of interest. We will show later that this problem can be eased and the resultant range sidelobe level is still acceptable in an attenuating medium. In particular, it will also be shown that chirp excitation is suitable for this pulse compression scheme.

It should be emphasized that the range sidelobe level reported in this paper is the overall compression result in an attenuating medium. Moreover, the shaping window only partially controls noise amplification associated with inverse filtering. If SNR improvement is critical, other filtering techniques, such as Wiener filtering, can be employed [5].

\section{SIGNALPROCESSINGANDEVALUATIONOF CODED SIGNALS}

\section{Inverse filtering in frequency domain}

Fig.1 illustrates the procedure of pulse compression using inverse filter in this paper. The inverse filter here is derived directly from the emitted coded signal. The shaping window is applied to control range sidelobes.

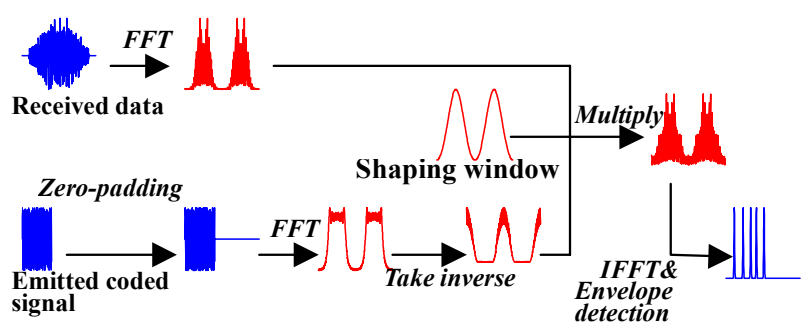

Fig. 1. Pulse compression scheme using inverse filter

\section{Effect of Incomplete echoes}

In general, the inverse filter produces no sidelobes if echoes are not truncated. Otherwise, the inverse filter can no longer compress these echoes perfectly. The resultant range sibelobes would arise and extend over the whole region of interest. Unfortunately, some incomplete echoes appear at the ends of received data, as 


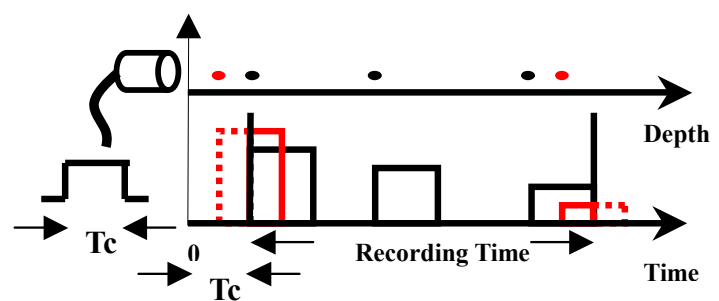

Fig. 2. Introducing of incomplete echoes (dashed-line)

illustrated in Fig. 2. In general, the first end comes from the fact that a transducer does not transmit and receive simultaneously. Thus, if a transducer is used for both transmit and receive, echoes from distances less than $\mathrm{Tc} 2 * \mathrm{c}$ cannot be received completely, where $\mathrm{c}$ and $\mathrm{Tc}$ denote the sound velocity and the duration of the coded signal, respectively. These incomplete echoes, if present, usually have large intensities and degrade the compression result greatly. To remove them, a non-scattering medium with a thickness of $\mathrm{Tc} / 2 * \mathrm{c}$ can be padded between the probe and the imaging body. An altemative is using different sets of transducers to transmit and receive, which is practicable especially in sparse transmit synthetic apeture systems [6] The latter, however, have to overcome the problem of inter-channel crosstalk.

Similarly, the incomplete echoes appear within the last $\mathrm{Tc}$ of the recording time. However, due to inherent attenuation in tissue, their levels will gradually reduce as the depth increases. With an attenuation of $0.5 \mathrm{~dB} / \mathrm{MHz} / \mathrm{cm}$ and center frequency of $5 \mathrm{MHz}$, for example, echoes from two depths of $15 \mathrm{~cm}$ and $20 \mathrm{~cm}$ are attenuated by $75 \mathrm{~dB}$ and $100 \mathrm{~dB}$, respectively. This implies that there is a trade-off between recording time and achievable range sidelobes. That is, the resultant range sibelobes can be controlled to an acceptable level simply by increasing the recording time, if necessary.

\section{Evaluation of coded signals}

Ideally, when inverse filtering is employed, the compression result is irrelevant to the coded signal. However, in the case of compressing the incomplete echoes, the behavior of range sibelobes appearing inevitably depends on the chosen coded signal. Fig. 3 shows a comparison of three compression results associated with chirp, pseudochirp, and random code excitations as all are identically truncated to have half of the original length at the last end. Random code was derived from a sequence of random numbers selected from a uniform distribution in the range [0, 1]; pseudochirp was a binary sequence derived from the continuous amplitude of chip [1], [2]. The result indicates that while the mainlobes still exist, their range sidelobes behave differently. For random code excitation, the range sidelobes act as an interference floor. In contrast, compressing truncated chirp results in lower range sidelobes. This is because for chip truncation in time domain has less effect on its spectrum shape, except that its bandwidth becomes smaller. But there are still some significant peak sidelobes folded into the beginning of the region of interest. A better result can be obtained by properly zero-padding the truncated signal before compression so as to remove them out of the region of interest. By doing so, compressing incomplete echoes for chirp excitation may only worsen the result around the truncated region. However, the effect of zero-padding on truncated random code is not significant because the resultant range sidelobes are uniformly distributed. The range sidelobes for pseudochirp excitation behave between the other two.

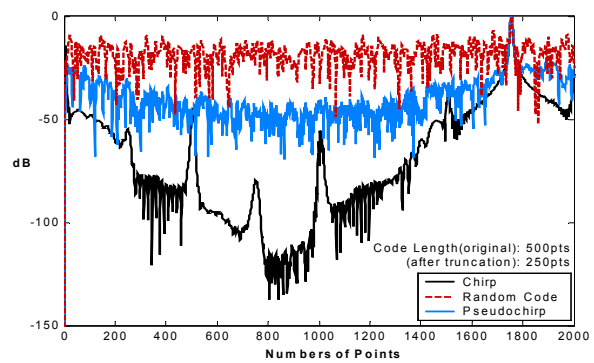

Fig. 3. Comparison of the compression results associated with three truncated coded waveforms

\section{Simulation Results}

To verify that in an attenuating medium, the proposed compression technique can perform well and the result can be further improved by increasing the recording time, three coded waveforms, including chip, pseudochirp, and random code with identical time duration of $25 \mu \mathrm{s}$, were investigated by simulations. Both chirp and pseudochip were centered at $5 \mathrm{MHz}$ with $5 \mathrm{MHz}$ bandwidth. The bit rate of random code was $20 \mathrm{MHz}$. Their corresponding spectra are shown in Fig.4. Point targets were located randomly over a range of $20 \mathrm{~cm}$ with average spacing of $0.375 \mathrm{~cm}$, as shown in Fig. 5. The attenuation coefficient of $0.5 \mathrm{~dB} / \mathrm{MHz} / \mathrm{cm}$ was considered. Assume that the combination of the transducer and the shaping window resulted in a Gaussian shape frequency response centered at $5 \mathrm{MHz}$ with $2.5 \mathrm{MHz}-6 \mathrm{~dB}$ bandwidth. Here, in order to evaluate the effects of incomplete echoes at the last end, the removal of the incomplete echoes at the first end was presumed. Fig. 6 (a) and (b) show the 

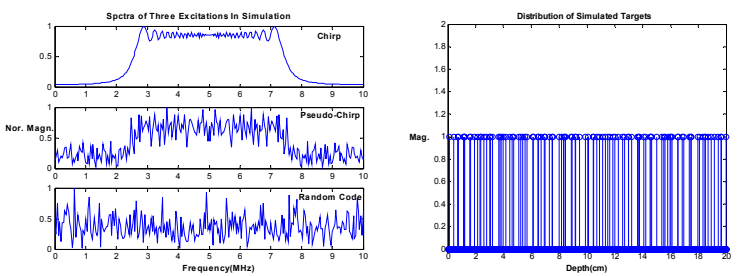

Fig. 4. Spectra of Three coded Fig. 5. Distribution of excitations in simulation simulated targets

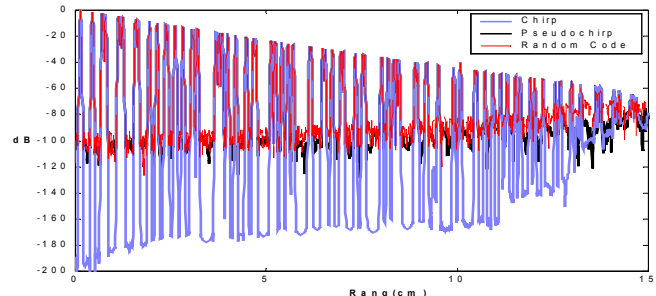

(a)

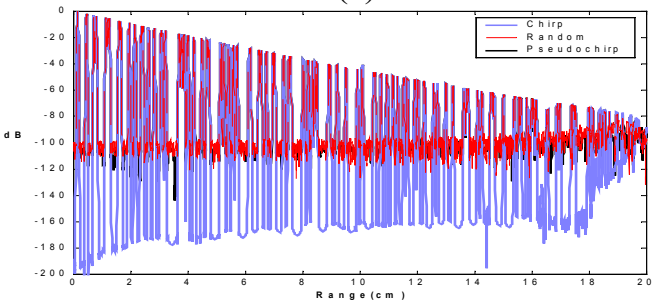

(b)

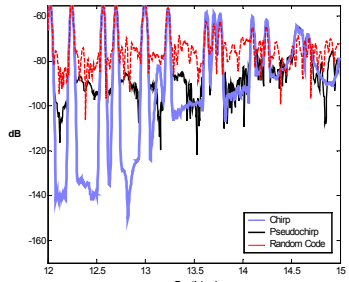

(c)

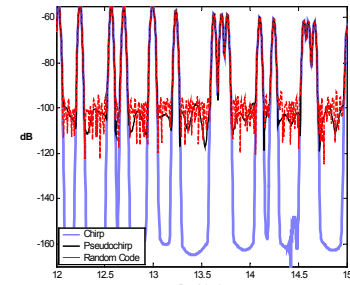

$(\mathrm{d})$
Fig. 6. The compression results in an attenuating medium when (a) data were recorded up to $15 \mathrm{~cm}$, (b) data were recorded up to $20 \mathrm{~cm}$, (c) zoom-in $(12 \sim 15 \mathrm{~cm})$ of (a), and (d) zoom-in (12 15cm) of (b).

compression results associated with three coded waveforms when the data were recorded up to a depth of $15 \mathrm{~cm}$ and $20 \mathrm{~cm}$, respectively. Fig. 6(c) and (d) show the zoom-in of (a) and (b) respectively. The results were normalized to their corresponding maximum responses. As evidenced in Fig.6, in an attenuating medium, the range sidelobes for three different excitations are at least below $-70 \mathrm{~dB}$. In particular, range sidelobes for chirp excitation are far lower than the others, and only arise at the last end. Except this region, as shown in Fig. 6(d), the range sidelobes are below-120dB. Comparing Fig.6(c) with(d), it is evident that when data are recorded up to a depth of $20 \mathrm{~cm}$ instead of $15 \mathrm{~cm}$, the levels of the range sidelobes for three excitations are greatly reduced.

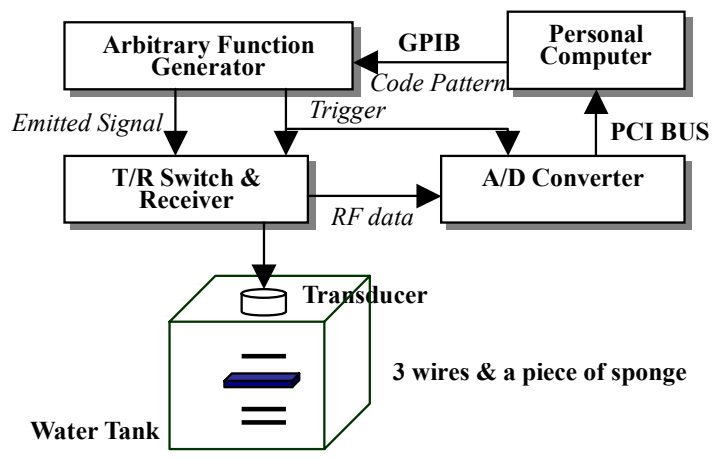

Fig. 7. Experiment setup

IV. Experimental Results

Pulse-echo experiments were conducted in a water tank which contains three wires and a piece of sponge for introducing attenuation. The experimental system is shown in Fig.7. A $5 \mathrm{MHz}$ transducer (V309, Panametrics) with focus at $8 \mathrm{~cm}$ was used. A $16 \mathrm{MHz}$ programmable arbitrary function generator (AFG320, SONY) was used to transmit pulse and the coded signals. The T/R switch \& receiver board has the functions of turning off the receiver during transmission and pre-amplifying the received data. A 12-bit $16 \mathrm{MHz}$ A/D converter(PCI-9812, ADLINK) was used to digitize the echoes. Three excitation waveforms with identical driving voltage of $4 \mathrm{~V}$, including pulse, pseudochirp and random code, were examined. Both pseudochirp centered at $4.4 \mathrm{MHz}$ with $4.4 \mathrm{MHz}$ bandwidth and random code were 400 points long (i.e., a length of $25 \mu \mathrm{s}$ ) while the pulse generated by the same function generator was just one point long. The first wire was placed far enough so that no incomplete echoes presented at the first end. Furthermore, received data for all excitations do not contain incomplete echoes at the last end. Fig. 8 and Fig. 9 show the received RF data and the comesponding compression results, respectively. In Fig.9, significant SNR improvements can be achieved by using coded excitations. Besides, the inverse filter for random code excitation causes more noise amplification than that for pseudochirp excitation.

The received RF data were then averaged over 10000 acquisitions to evaluate the achievable range sidelobes. The compression result shown in Fig. 10 indicates that the sidelobe levels for all excitations 
are about $60-70 \mathrm{~dB}$ below the maximum responses and only slightly differ, which implies that in our experimental system, there still exists some interference that cannot be averaged out. A lower range sidelobe level for coded excitation may be achievable if such interference can be suppressed. The effect of compressing incomplete echoes can be observed in Fig.11. Here, the recording time was shortened so that the echoes from the last two wires were truncated. Again, as demonstrated in simulation results, range sidelobes for both excitations obviously increase, but the last two wires can still be recognized. Moreover, pseudochirp excitation indeed outperforms random code excitation.

\section{V.CONCLUSION}

In this paper, attenuation estimation is dismissed. By implementing the pulse compression with inverse filtering in frequency domain instead of in time domain, it has been shown that in an attenuating medium, lower range sidelobes still can be obtained by increasing the recording time. Furthermore, chirp excitation was shown to be suitable for this scheme. However, there are still some realistic problems associated with this scheme: (1) the removal of incomplete echoes at the first end may be practicable only when dedicated transducers for receiving are employed, and (2) because the data recording time should be long enough to reduce the effect of incomplete echoes on range sidelobes, the system complexity is higher than other conventional methods.

\section{REFERENCES}

[1] M. O'Donnell, 'Coded excitation system for improving the penetration of real-time phased-array imaging systems," IEEE Trans. Ultrason., Ferroelect., Freq. Contr., vol.39, pp. 341-351, May 1992.

[2] P. -C. Li, E. Ebbini, and M. O’Donnell, "A new filter design technique for coded excitation systems," IEEE Trans. Ultrason., Ferroelect., Freq. Contr., vol.39, pp. 693-699, Nov. 1992.

[3] K. Eck, R. Schwann, A. R. Brenner, and T. G Noll, "Depth-dependent mismatched filtering using ultrasonic attenuation as a filter design parameter," Proc. IEEE Ultrason. Symp., 1998, pp.1639-1644.

[4] T. X. Missaridis, M. H. Pederson, and J. A. Jensen, 'Clinical use and evaluation of coded excitation in B-mode images," Proc.
IEEE Ultrason. Symp., 2000, pp.1689-1693.

[5] B. Haider, P. A. Lewin, and K. E. Thomenius, "Pulse elongation and deconvolution filtering for medical ultrasonic imaging," IEEE Trans. Ultrason., Ferroelect., Freq. Contr., vol.45, pp. 98-113, Jan. 1998.

[6] R. Y. Chiao, and L. J. Thomas, “ Synthetic transmit aperture imaging using orthogonal Golay coded excitation," Proc. IEEE Ultrason. Simp.. 2000. pv.1677-1680.

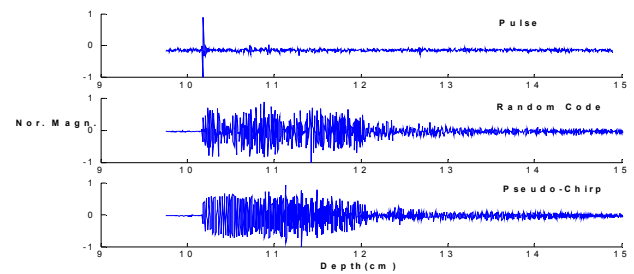

Fig. 8. Received RF data for pulse (top), random code (center) and pseudochirp (bottom) excitation

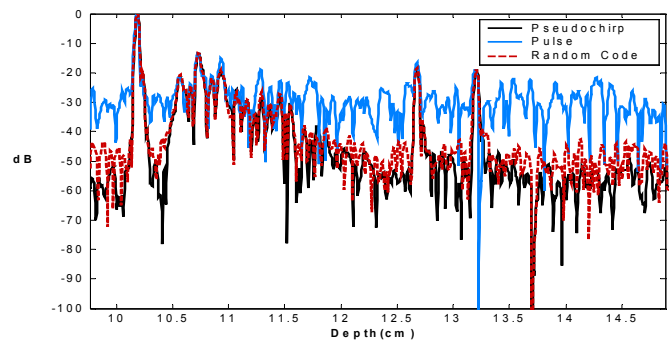

Fig. 9. Compression results for three excitations (Received RF data were not averaged)

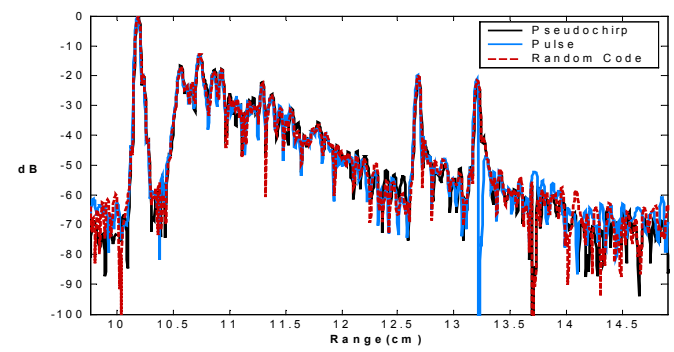

Fig. 10. Compression results for three excitations (Received RF data were averaged)

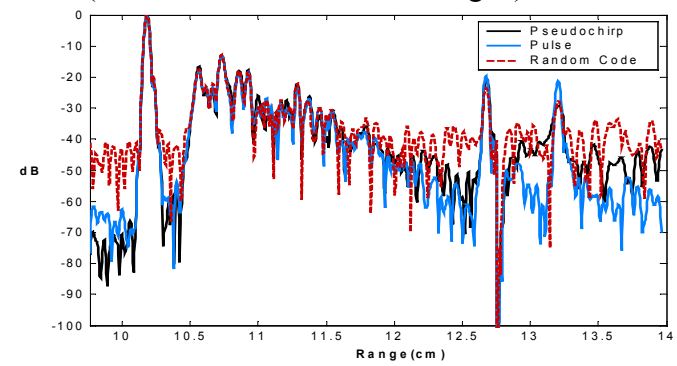

Fig. 11. Compression results for three excitations when last two wires were truncated (RF data were averaged) 